\title{
Criminologie
}

\section{Un rappel historique de la libération conditionnelle : deux volets d'une évolution}

\section{Michel Nicolas}

Volume 14, numéro 2, 1981

Libération conditionnelle : évolution et application (1899-1981)

URI : https://id.erudit.org/iderudit/017141ar

DOI : https://doi.org/10.7202/017141ar

Aller au sommaire du numéro

Éditeur(s)

Les Presses de l'Université de Montréal

ISSN

0316-0041 (imprimé)

1492-1367 (numérique)

Découvrir la revue

Citer cet article

Nicolas, M. (1981). Un rappel historique de la libération conditionnelle : deux volets d'une évolution. Criminologie, 14(2), 73-80.

https://doi.org/10.7202/017141ar d'utilisation que vous pouvez consulter en ligne.

https://apropos.erudit.org/fr/usagers/politique-dutilisation/ 


\section{UN RAPPEL HISTORIQUE DE LA LIBERATION CONDITIONNELLE : DEUX VOLETS D'UNE ÉVOLUTION}

Michel Nicolas

L'intention de la libération conditionnelle remonte déjà à plus d'un siècle! Depuis son origine jusqu'à nos jours le système des libérations conditionnelles a connu, par vagues successives, un essort considérable tant sur le plan du raffinement des méthodes de libération que sur le plan strictement quantitatif des personnes soumises au système.

À n'en pas douter le régime canadien des libérations conditionnelles a grandement changé et nous voudrions rappeler les grandes lignes de cette évolution. Toutefois, le rayonnement du système des libérations conditionnelles ne doit pas être un obstacle à une brève analyse de l'évolution de la nature même de la libération conditionnelle.

En effet, peut-on parler d'une aussi grande évolution de la nature crimino-juridique de cette trouvaille sociale qu'est la mise en liberté sous condition des détenus?

\section{LE RÉGIME DES LIBÉRATIONS CONDITIONNELLES}

\section{A) L'origine}

L'idée de la libération conditionnelle prend sa source dans un système de pardon instauré en Australie à la fin du xvirie siècle. Ce système permettait alors au gouverneur de ces colonies pénitentiaires britanniques de mettre fin à la détention de prisonniers ayant fait preuve de bonne conduite et de bonne volonté dans leur travail.

Rapidement au début du Xix ${ }^{e}$ siècle, sous l'égide du gouverneur Alexandre Maconochie, le système se raffine de façon à favoriser la liberté des détenus ayant accumulé un certain nombre de points leur permettant d'acheter leur «ticket of leave».

Au milieu du XIX ${ }^{e}$ siècle Sir Walter Crofton, directeur d'une prison irlandaise, reprend l'idée d'un système de pointage mais il le greffe à un mode graduel de libération : entre le cachot et l'air libre le détenu devait traverser les paliers du travail et de la prison à sécurité minimale. 
À cette même période et sous l'influence de Crofton, un élément essentiel du système fait son apparition : le libéré est confié à la police qui doit l'assister et le surveiller.

Vers la fin du $\mathrm{XIX}^{\mathfrak{e}}$ siècle ce nouveau courant a déjà traversé l'Europe et les États-Unis de sorte qu'un système progressif de libération devient pour les réformateurs une nécessité pénologique.

B) L'évolution du régime canadien des libérations conditionnelles

Le rapport du comité sénatoriel (1974) portant sur la libération conditionnelle rappelle les trois grandes étapes du régime canadien :

- avant 1899 , la prérogative royale de clémence;

- de 1899 à 1958, Ia loi sur la libération conditionnelle;

- à partir de 1958, une réforme complète de la loi instituant, en outre, une commission des libérations conditionnelles.

Depuis la parution de ce rapport le régime s'est enrichi d'une quatrième étape importante :

- dès 1977 , la loi canadienne permet d'une façon formelle la création de commissions provinciales.

Avant 1899, la libération résulte de l'exercice de la prérogative royale de clémence. Le nombre de libérés est alors minime et selon le rapport du Sénat, elle est généralement inconditionnelle, étant donné l'absence de surveillants.

En 1899, la première loi des libérations, copie du Penal Servitude Act de l'Angleterre, permet un élargissement avant la fin de la sentence à la suite de l'avis d'un fonctionnaire. En 1913, le service des pardons voit le jour et on lui confie la tâche d'enquêter sur les cas des demandes de libération. Selon le rapport Archambault (1938) «trois fonctionnaires empruntés à la Royale Gendarmerie à cheval du Canada » assistent le chef du service des pardons dans ses enquêtes. En 1949, le service ouvre des bureaux à Montréal et à Vancouver.

De 1899 à 1958 , le nombre des libérations est toujours très limité. Selon le rapport Fauteux (1956) le nombre de libérés est de 778 en 1930, et de 942 en 1949.

En 1958, à la suite des recommandations du rapport Fauteux (1956) la loi actuelle est adoptée. Cette loi crée une commission chargée d'accorder la libération conditionnelle et de la révoquer 
au besoin. Le service des libérations conditionnelles proposé dans le même rapport est simultanément mis sur pied, et ce, dans le but de procéder aux enquêtes de pré-libération et de surveiller les détenus libérés sous condition.

Â partir de ce jour, le nombre des libérés conditionnels augmente sensiblement. Le groupe d'étude mandaté en 1972, sous la présidence du juge Hugessen, pour étudier l'organisation et les méthodes appliquées en matière de libération conditionnelle, cite dans son rapport (1973) que le nombre de libérés dépasse 2000 en 1960,3000 en 1968 et 6000 en 1971 .

Finalement en 1977, le Parlement fédéral légifère afin de permettre la création de commissions provinciales ayant juridiction sur toutes les personnes détenues dans les institutions carcérales provinciales. Soulignons que l'année suivante le Québec créait cette commission et que le Service de probation se voyait confier la surveillance de ses libérés.

En 1979, il y avait plus de 7000 personnes surveillées par le système fédéral tandis que celui du Québec avait déjà près de 700 libérés de ses propres institutions carcérales. Le régime se porte bien!

2. L'ÉVOLUTION DE LA NATURE CRIMINO-JURIDIQUe DE LA LIBÉRATION CONDITIONNELLE

Pascal disait : On peut bien connaître l'existence d'une chose, sans connaître sa nature. " Dans le cas d'une créature pénologique telle la libération conditionnelle, il faut bien dire qu'un effort de reconnaissance apparaît essentiel pour tout au moins en justifier l'existence.

\section{A) La nature criminologique}

En principe de loi canadienne de 1889 , copiée sur le Penal Servitude Act, devait préconiser une attitude plus positive face aux personnes incarcérées. En Angleterre, cette loi permettait ainsi d'incorporer la libération conditionnelle au système progressif mis de l'avant dans les prisons (cellule - travail forcé - travail industriel - libération conditionnelle). Inscrite dans ce processus, la libération devient l'ultime stimulus favorisant la collaboration du prisonnier. La libération devient alors une nette mesure de contrôle de la population pénitentiaire selon le discours tenu par Sir Walter Crofton au Congrès du Cincinnati (1870).

Toutefois, Hawkins (1971) soutient que le véritable motif du Penal Servitude Act était plutôt de réduire le nombre de 
personnes emprisonnées afin de pouvoir solutionner les problèmes de surpopulation des institutions carcérales. L'essence de la libération consiste alors à régulariser le flot des prisonniers, encore qu'elle soit tempérée par l'aspect intimidant de la loi qui autorise la révocation de la libération conditionnelle.

Au Canada, la réalité est tout autre; la surpopulation carcérale n'est pas un problème au début du siècle et le «mark system », ou système progressif, n'existe pas. Pourtant on copie le Penal Servitude Act!

La raison d'être de la libération se découvre à travers les paroles du Premier ministre canadien de l'époque, Sir MacDonald :

Voici au pénitencier, par exemple, un jeune homme de bon caractère qui peut avoir commis un crime dans un moment de passion, ou être tombé victime peut-être, du mauvais exemple, ou de l'influence de mauvais amis. Depuis son incarcération les rapports sur son compte sont favorables ...

Pendant 70 ans (1889-1959) la nature de la libération conditionnelle canadienne oscille entre le pardon-récompense et l'humanisme gratuit et influent. La libération est d'autant plus un cadeau qu'il n'existe à peu près pas de mesure d'aide ou de contrôle des personnes libérées conditionnellement.

Sous l'impulsion de la Commission Fauteux (1956), la nature de la libération conditionnelle change radicalement. De 1959 à 1970 elle est une mesure sélective à caractère scientifique, visant la protection de la société et la réhabilitation du détenu. Le libéré est lobjet d'une évaluation psycho-sociale, d'une décision administrative, d'une libération avec conditions sous la surveillance d'agents du service des libérations, instituté en 1959.

La sélection se veut non plus assujettie au seul aspect qu'une bonne conduite peut être récompensée mais plutôt fondée sur une appréciation soigneuse d'éléments tels :

Les progrès du détenu dans l'institution y compris sa participation au programme de formation professionnelle ou académique; son changement d'attitude par suite de son expérience comme détenu; ses chances de réadaptation au foyer, au travail et dans la collectivité; et sa volonté de

1. Extrait du rapport Fauteux, 1956, p. 58. 
mériter son élargissement et d'accepter l'aide de ses surveillants ${ }^{2}$.

Près d'un siècle plus tard on retrouve au Canada l'idée originale du Mark System de Crofton, au sens où l'on évalue à travers une série de critères les chances de succès d'une libération éventuelle. Il faut néanmoins établir une distinction fondamentale entre l'idée de Crofton et le concept de libération préconisé par Fauteux : la libération conditionnelle est en elle-même une formule de resocialisation pour certains élus et non pas l'aboutissement d'un système progressif ainsi que l'envisageait Crofton. À partir du rapport Fauteux le regard ne porte plus seulement sur l'intérieur, soit sur le comportement en prison, mais autant sur l'extérieur, c'est-à-dire sur des éléments favorisant le retour du détenu à une vie de citoyen.

La libération devient un baume destiné aux personnes choisies. Les effets curatifs d'une liberté sous contrôle justifient l'existence du système. La définition de la libération conditionnelle que donne un juge de la Cour suprême de l'Utah illustre à la fois la conception américaine et canadienne des années soixante :

La libération conditionnelle est une mesure de réforme fondée par une stratégie et une politique d'aide afin de lui permettre de résister à la tentation, de se maîtriser, d'ajuster ses attitudes et sa conduite aux normes sociales; la libération conditionnelle vise à lui donner plus de liberté et de possibilités de vivre normalement à l'intérieur du corps social au fur et à mesure que sa volonté de faire face à ses responsabilités s'affermit (Traduction : McCoy $v$ Harris, 108 Utah 407).

Au Canada, le début des années '70 marque un point tournant quant à la nature criminologique de la libération conditionnelle. En effet, ces années voient naître la mise en vigueur du régime de surveillance obligatoire proposé par le rapport Ouimet (1969). La surveillance obligatoire est ni plus ni moins une libération conditionnelle obligatoire. C'est la consécration des effets bénéfiques d'une libération surveillée. La libération ne repose plus seulement sur un choix judicieux des candidats pouvant bénéficier de la mesure mais se justifie aussi par la nécessité d'une étape de transition, étape au cours de laquelle les ex-détenus jouissent de l'aide, de l'assistance et de la surveillance des agents de l'État. L'argumentation est simple : les détenus non sélection-

2. Fauteux, 1956, p. 55-56. 
nés pour une libération conditionnelle ont autant besoin de cette aide et de cette surveillance que ceux qui l'ont été.

On peut conclure que la nature de la libération conditionnelle canadienne, tout en demeurant un instrument pénologique, a connu trois grandes étapes :

1) elle a d'abord été un instrument de récompense pour ceux des détenus qui offraient une bonne collaboration;

2) elle a ensuite été un instrument d'aide pour ceux des détenus qui semblaient devoir être aidés, assistés et surveillés;

3) elle a enfin été un instrument de transition, partant de l'idée de la nécessité d'un retour surveillé du détenu à la vie de citoyen.

\section{B) La nature juridique}

Malgré l'évolution de la nature criminologique de la libération conditionnelle, sa nature juridique demeure immuable : la libération conditionnelle est un privilège. Même à la suite de la mutation importante proposée par Fauteux et concrétisée par la loi de 1959, la Cour suprême, dans l'arrêt McCaud, réitère la nature juridique de la libération conditionnelle en ces termes :

Le fait de purger sa sentence en prison ou en libération conditionnelle est une décision qui relève du pouvoir discrétionnaire de la Commission des libérations conditionnelles (Ex Parte McCaud, 1965, 1 C.C.C. 168).

Mais les tribunaux prennent conscience que la libération conditionnelle n'est plus une récompense ou un Acte de clémence qui touche quelques heureux bénéficiaires.

À partir de 1959 le nombre de libérés augmente considérablement, et ils sont davantage surveillés. La Commission et le service des libérations conditionnelles prennent des décisions (libération, suspension, révocation) lourdes de conséquences. Devant cet état de fait les tribunaux deviennent plus critiques face aux décisions.

En 1965 : L'arrêt McCaud note que les décisions prises en ce domaine doivent répondre à l'exigence d'agir d'une manière équitable.

En 1974 : Le juge Dickson souligne dans l'arrêt Howarth v N.P.B. (Cour suprême, le 11 octobre 1974) "qu'il ne peut vraiment pas voir comment on peut prétendre que la libération conditionnelle 
est un simple privilège ...» $(1976,1$ R.C.S. 453 ).

En 1975 : Le juge en chef Laskin déclare dans l'arrêt Mitchell $v R$. (Cour suprême, le 7 octobre 1975) que «la Commission des libérations conditionnelles se réclame d'un pouvoir tyrannique qui est sans précédent à l'intérieur d'organismes administratifs qui touchent à la liberté des personnes. Elle réclame un pouvoir sans entrave de surveiller le détenu tout comme s'il était une marionnette au bout d'une ficelle» (traduction).

La nature juridique de la libération conditionnelle demeure un privilège, mais les décisions de libérer, de suspendre, de révoquer sont et seront l'objet d'analyses beaucoup plus critiques.

3. L'AVENIR DE LA LIBÉRATION CONDITIONNELLE

Aujourd'hui le système des libérations conditionnelles prospère. Il est fédéral et provincial. Il est régionalisé et sectorisé. On pourrait même prétendre qu'il est fortement «bureaucratisé ».

Malgré cette prospérité, nous croyons qu'il y a discordance entre d'une part la nature pénologique et juridique de la libération conditionnelle et d'autre part l'existence même du système actuel, et que cette discordance mérite un temps d'arrêt, un moment de réflexion.

En effet, pourra-t-on prétendre longtemps que la libération conditionnelle est un privilège si la presque totalité des détenus jouissent (ou subissent, selon le point de vue) d'une libération conditionnelle ordinaire ou obligatoire?

Pourra-t-on affirmer longtemps que la libération surveillée est une étape de transition nécessaire mais en même temps un privilège soumis à un pouvoir discrétionnaire obscur?

Peut-on parler d'étape de transition lorsque des personnes sont libérées sous surveillance après avoir purgé moins d'un an d'incarcération?

Bientôt, si on ne remet pas en question l'existence même du système, il faudra tout au moins s'intéresser à la nature de la libération conditionnelle puisque de toute façon inévitablement sa manière d'exister ne peut plus rester dans l'ombre.

Le système n'aura pas à répondre seulement de certaines de ses décisions devant les tribunaux : il devra faire face à sa réalité quotidienne, réalité qui sera de plus en plus l'objet d'analyses 
sociologiques telle cette magistrale étude de McCleary dans son livre Dangerous Men : The Sociology of Parole (1978).

Dans l'avant-propos de ce livre, David Fogel écrit : [...] a more functionally efficient parole bureaucracy will not lead to a more fundamentally sound or rational system of parole [...] [...] it is the fundamentally unjust nature of parole which leads inevitably to dysfunction in the organization charged with parole supervision (p. 12).

\section{BIBLIOGRAPHIE}

COMMISSION DE RÉFORME DU DROIT DU CANADA (1976), Études sur l'emprisonnement, Approvisionnements et Services Canada, Ottawa.

HAWKINS, Keith (1971), * Parole Selection : The American Experience *, Ph.D. Thesis University of Cambridge.

McClEARY, Richard (1978), Dangerous Men : The Sociology of Parole, Sage Publication California.

RAPPORT ARCHAMBAULT (1938), Rapport de la Commission royale d'enquête sur le système pénal du Canada, Imprimeur du Roi, Ottawa.

RAPPORT FAUTEUX (1956), Rapport d'un comité institué pour faire enquête sur les principes et les méthodes suivis au Service des pardons du ministère de la Justice du Canada, Imprimeur de la Reine, Ottawa.

RAPPORT GOLDENBERG (1974), Rapport du comité sénatoriel permanent des affaires juridiques et constitutionnelles, la libération conditionnelle au Canada, Information Canada, Ottawa.

RAPPORT HUGESSEN (1973), Rapport du groupe d'étude sur la mise en liberté des détenus, Information Canada, Ottawa.

RAPPORT OUIMET (1969), Rapport du Comité canadien de la réforme pénale et correctionnelle, Imprimeur de la Reine, Ottawa.

Transactions of the National Congress on Penitentiary and Reformatory Discipline (1871, Ed. E.C. Wines, Albany. 